\title{
Délits et peines militaires à Rome sous la République: desertio et transfugium pendant les guerres civiles
}

\author{
Mariama GuEYE \\ Université Chekh Anta Diop (Dakar) \\ mariamagueye@yahoo.com
}

Recibido: 15 de mayo 2012

Aceptado: 28 de junio 2013

\section{RÉSUMÉ}

Cette étude analyse l'application effective des sanctions réservées au miles en cas de désertion. Dans le code pénal militaire romain, la désertion constitue un délit militaire grave passible de la peine de mort. Pourtant, les sanctions très sévères habituellement réservées aux déserteurs et transfuges de l'armée romaine sont rarement voire exceptionnellement mises en application en période de bellum intestinum. En marchandant la fidélité des soldats, les chefs de l'armée romaine encouragent et orchestrent ouvertement leur désertion. Dans le contexte chaotique des conflits civils, la desertion est alors utilisée comme une arme militaire et politique pour affaiblir ou anéantir l'adversaire.

Mots clés: Châtiments. Délit militaire. Desertio. Guerre civile. Perfugium. République. Sacramentum. Transfugium.

\section{Offenses and military sanctions in Rome under the Republic: desertio and transfugium during the civil wars}

\begin{abstract}
This study analyzes the effective application of sanctions reserved for miles in case of desertion.In the Roman military penal code, the desertion constitutes a grave military offense liable to the death penalty. However, severe sanctions usually reserved for deserters and defectors from the Roman army are rarely or exceptionally applied during intestinum bellum. By haggling over the loyalty of the soldiers, the leaders of the Roman army encourage and orchestrate openly their desertion. In the chaotic context of the civil conflicts, desertion is then used as a military and political weapon to weaken or annihilate the opponent.
\end{abstract}

Key words: Civil war. Desertio. Military offense. Perfugium. Punishment. Republic. Sacramentum. Transfugium.

Sommaire: 1. Introduction. 2. Les contrevenants au réglement militaire: le desertor, le perfuga et le transfuga. 3. Châtiments réservés au desertor et au transfuga. 4. Fréquence et impunité du délit du desertio et de transfugium. 5. La desertion utilisée comme une arme militaire et politique. 


\section{Introduction}

Selon Tite Live, ${ }^{1}$ la désertion ${ }^{2}$ est un délit passible de la peine de mort à Rome. Considérée comme une violation du règlement militaire, elle reste durement sanctionnée sous la République. Déserteurs et transfuges s'exposent à des châtiments qui vont de la flagellation à la crucifixion en passant par la précipitation du haut de la roche tarpéienne. Pourtant, en dépit de ces peines très lourdes prévues, des mouvements de désertion individuels ou collectifs se produisent constamment au cours des guerres civiles. L'étude de La guerre civile, ${ }^{3}$ la Guerre d'Afrique ${ }^{4}$ de César et des Guerres civiles à Rome ${ }^{5} \mathrm{~d}$ 'Appien révèle d'une part la fréquence impressionnante des cas de désertions, d'autre part la quasi-absence de châtiments des coupables.

L'objet de cet article est de tenter de montrer que, malgré les sanctions très sévères et dissuasives traditionnellement réservées aux déserteurs et transfuges ${ }^{6}$ dans l'armée romaine, en période de guerre civile, la désertion reste une infraction mineure presque impunie. Toutefois, la desertio peut-elle constituer un délit majeur dans un contexte où la plupart des règles censitaires et religieuses sur lesquelles reposent les valeurs fondamentales de l'armée romaine, telles que la discipline, la fidélité ou le respect du sacramentum sont ouvertement bafouées? Est-il d'ailleurs concrètement possible d'appliquer les sanctions prévues en cas de désertion dans une situation chaotique où le fonctionnement "normal" de l'armée est suspendu?

Dans un premier temps, il convient de définir les notions de desertor, de perfuga et de transfuga pour mieux cerner ces vocables usités quelquefois avec la volonté d'afficher la supériorité de son camp et les types de châtiments infligés habituellement aux transfuges et déserteurs au cours des guerres extérieures afin de mettre en relief leur absence pendant les dissensions civiles. L'analyse des cas précis de transfuges nous permettra d'interroger leur utilisation multiple dans les situations complexes que créent les guerres civiles pour tenter d'éclairer l'attitude des responsables militaires et politiques.

1 Il ajoute qu' "on avait vu des pères sanctionner cette loi par la mort même de leurs enfants" (Liv. XXIV,39). Polybe le note déjà en indiquant que la mort était la sanction infligée à celui qui abandonnait son rang (Plb. I,17,11).

2 Déserteurs et transfuges ont déjà fait l'objet de nombreuses études parmi lesquelles on peut citer celles de M. Vallejo Girvés et de C. Wolff. M. Vallejo Girvés a abordé la répression de la désertion dans VALLEJO GIRVÉs 1993, 241-251, avant de mettre l'accent sur les édits destinés à lutter contre la désertion dans l'armée impériale dans VALLEJo GIRVÉs 1996, 31-47 et d'analyser plus largement les causes et les conséquences des désertions dans VALLejo GiRvés 1996a, 399-408. Quant à C. Wolff, après avoir tenté de dégager le profil des transfuges et des déserteurs, dans WolfF 2009, elle a analysé les conditions dans lesquelles intervenaient ces désertions. Enfin, il faut signaler en dernier lieu l'article de P. Cosme 2003, 287-307. Dans le monde grec on peut citer l'étude de G. Velho dans laquelle il montre l'image négative que la société grecque avait du déserteur, cet “anti-citoyen",VELHO 2002, 239-256.

3 Caes., BC, I,20-23; I,76; II,28; III,13; III,61; III,71.

4 On retrouve également de nombreux cas de transfugium et de perfugium dans le Pseudo-César, $B A, 8,5$; 19,$1 ; 32,3 ; 35,2-3 ; 55,1 ; 66,1 ; 66,4 ; 74,1-2$.

5 App., BC, I,10,85-91; III,44; III,47-48; III, 56; V,17; V,38; V,80, V,96; V,100; V,102; V,125; V,142.

6 Il convient de préciser que la Loi des Douze Tables, du moins ce qu'il en reste, ne prévoit pas de sanction précise à l'endroit du déserteur. Ce n'est qu'à partir de la période impériale que les textes juridiques définissent et fixent clairement les peines réservées aux déserteurs. Toutefois Tite-Live, Valère-Maxime, Plutarque mentionnent les châtiments infligés aux déserteurs sous la République, cf. infra. 


\section{Les contrevenants au règlement militaire: le desertor, le perfuga et le transfuga}

Dans le vocabulaire militaire latin, trois termes sont fréquemment employés pour désigner celui qui quitte volontairement l'armée sans permission: ${ }^{7}$ desertor,${ }^{8}$ perfuga ${ }^{9}$ et transfuga. ${ }^{10}$

Ces vocables qui indiquent une catégorie de contrevenants au règlement de l'armée sont utilisés habilement par César dans sa stratégie démonstrative afin de mettre en évidence la supériorité de son armée. Par définition, le desertor est le soldat qui, sans congé, ${ }^{11}$ s'est éloigné de son camp, ${ }^{12}$ abandonnant ainsi volontairement son poste ou le corps dans lequel il est intégré. ${ }^{13} \mathrm{Il}$ est, par conséquent, coupable d'un délit militaire:

Omne delictum est militis, quod aliter quam disciplina communis exigit: veluti segnitiae crimen, vel contumaciae, vel desidiae. ${ }^{14}$

Quant au transfuge, transfuga ou perfuga, il désigne "l'homme libre ou l'esclave qui est passé à l'ennemi par sa volonté". ${ }^{15}$ Mais, à la différence du transfuga, le perfuga est, quant à lui, "un suppliant" qui cherche refuge auprès de l'ennemi. Toutefois, il faut distinguer le desertor du transfuga en dépit de ce que postule Isidore de Séville lorsqu'il précise que qui deserunt exercitum ad hostes transeuntes et ipsi desertores vocantur, ${ }^{16}$ car les crimes de desertio et de transfugium ne se situent pas au même niveau. En effet, si le desertor est la personne qui a quitté les rangs de son unité sans permission, le transfuge est, quant à lui, le soldat qui, animé par "l'espoir d'obtenir des avantages", ${ }^{17}$ passe volontairement à l'ennemi. Cette définition de Pos-

7 Toutefois, "le soldat en congé n'est pas censé être absent pour la République” (Dig., XLIX,16,1). Mais précisons, ici, qu'il s'agit bien d'un congé motivé ou honorable au lieu d'un congé infâmant.

8 Caes., BC, II,32; Sen., Clement., I,16,3.

9 Caes., BC, I,78; II,18; II,27; II,28; II,38; II,39; III,49; III,61; III,63; III,71; III,79; III,84. Liv. XXIV,20.

10 Liv., Per., LI, LV; Val. Max. II,7,11; II,7,12.

11 Glare 1982, s.v. Desertor.

12 Cet éloignement est perçu comme une désertion dès lors que le soldat se trouvait hors de la portée des sons des trompettes (Daremberg - Saglio, 1877-1919, s.v. Desertor). "The Roman army regarded as a desertor anyone who did not appear at roll-call" (LE BoHEc 2004). Dans ses articles 398 et 401, le code de la justice militaire français reprend l'absence sans autorisation dans sa définition du déserteur en temps de paix comme en temps de guerre (Code, Des peines applicables par les juridictions des forces armées et des infractions d'ordre militaire Titre II).

13 "Les déserteurs tiennent leur nom de ce qu'ils s'échappent, désertant le devoir militaire" (Isid., Etym., IX,3,39).

14 "Le délit militaire est tout ce qui porte atteinte à la discipline commune, comme la lâcheté, la rébellion et la paresse" (Dig., XLIX,16,6).

15 Perfugam qui liber, aut servus, aut hostes sui voluntate ad hostes transierit, qui idem dicitur transfuga (Festus, s.v. Perfuga).

16 “On appelle aussi déserteurs ceux qui désertent l'armée pour passer à l'ennemi" (Etym., IX,3,39). Nous ignorons à quelle date remonte cette extension de sens. Cosme $(2003,291)$ cite ce passage d'Isidore de Séville sans fournir de précision.

17 Le perfuga n'est pas tant l'individu qui fuit d'autres hommes, que celui qui fuit vers quelqu'un dans l'espoir d'obtenir quelque avantage", "perfugam esse non tam qui alios fugiat, quam qui ob spem commodorum 
tumius Festus sied parfaitement au transfuge des guerres civiles. En effet, en rejoignant le camp adverse, le transfuge ne quitte pas l'armée romaine, ce qui rend la trahison moins flagrante, mais change plutôt de commandant en chef afin d'obtenir plus de gratifications. En revanche, le déserteur n'est ni un réfractaire, ${ }^{18}$ ni un emansor. ${ }^{19}$ Quant au transfuge, il est non seulement un desertor mais également un proditor. En vérité, il commet un double délit: celui de désertion et celui de trahison. Le second crime génère automatiquement des circonstances aggravantes.

Dans La guerre civile ${ }^{20}$ et la Guerre d'Afrique, ${ }^{21}$ César utilise presque systématiquement perfuga et moins souvent desertor. Ce constat atteste que pendant la guerre civile le délit de transfugium, plus grave que celui de desertio, demeure très courant. L'emploi fréquent de perfuga permet à César d'afficher la supériorité de ses forces, présage de sa victoire. De fait, contrairement à desertor (qui désigne le soldat qui quitte son poste sans une destination précise), le vocable perfuga, quant à lui, indique clairement que le déserteur vient se refugier chez l'ennemi. Par conséquent, son emploi répété fait ressortir d'une part la forte domination du camp des Césariens où affluent massivement les soldats pompéiens qui viennent y chercher refuge et proposer simultanément leurs services, d'autre part l'affaiblissement progressif du camp adverse. D'ailleurs à Dyrrachium, César le dit lui-même lorsqu'il relève qu'il est rare de voir des milites le quitter pour Pompée alors que, tous les jours des hommes du camp de Pompée viennent vers César. ${ }^{22}$ En Afrique également chaque jour, des déserteurs gétules et numides provenant du camp de Scipion rejoignent en masse César. ${ }^{23}$ Il se sert de ces désertions massives qui se produisent dans le camp des Pompéiens pour annoncer l'imminence de la défaite de l'ennemi qui perd progressivement ses forces.

ad quempiam perfugiat (Festus, s.v. Perfuga).

18 Le réfractaire est le soldat qui, inscrit sur le cens, ne répond pas à l'appel de l'armée. Sur les motifs justifiant l'absence du soldat enrôlé, cf. Aulu-Gelle, Noct. Att., XVI, 4. Toutefois, le desertor devient un defector lorsqu'il abandonne son chef ainsi que sa cause. En octobre et novembre 44, les soldats qui ont quitté le camp d'Antoine sont non seulement des desertores mais aussi des defectores puisqu'ils lâchent simultanément la cause et leur chef à qui ils reprochent de ne pas venger la mort de César (App., $B C$, III,40,164; III,43,175).

19 Quant à l'emansor, c'est le soldat qui s'éloigne du camp momentanément mais avec l'intention d'y retourner. Il n'est qu'un vagabond, emansor est, qui diu vagatus ad castra regreditur (Dig., XLIX,16,3,2). Mais ce n'est qu'à partir de l'époque impériale que cette distinction devient plus précise. En effet, le nombre de jours pendant lesquels le soldat s'est absenté ainsi que la distance parcourue sont clairement définis dans le Digeste ce qui permet de déterminer la peine réservée au coupable (Dig., XLIX,16,4,13-14; Cosme 2003, 300303). Il faut préciser que le desertor ne devient pas proditor, tant qu'il n'est pas passé à l'ennemi.

20 Dans La guerre civile, César emploie au moins 17 fois perfuga et sa forme verbale perfugere (II,39,2; II,38,1; III,71,4; II,28,2; III,49,2; III,63,5; I,78,2; II,38,1; II,23,3; II,39,2; III,84,5; III,79,6; II,18,3; III,60,4; II,27;1; III,49,2; III,61,2) alors que desertor et deserere reviennent à 9 reprises (II, 32,7; II, 32,8; II,30,2; III,13,3; III,18,1; I,13,4; I,15,3; I,76,2; I,75,2).

21 Il n'utilise pratiquement que perfuga (perfugere): 8,$5 ; 19,1 ; 32,3 ; 35,2-3 ; 35,4-5 ; 55,1 ; 56,2 ; 62,5 ; 66,1$; 68,2 .

22 Caes., $B C$, III,61,2.

23 Caes., $B A, 32,3$. 


\section{Châtiments réservés au desertor et au transfuga}

La désertion, marque extrême d'insoumission et de lâcheté pouvant mettre en péril l'organisation et la survie des composantes de l'armée, était punie de mort ${ }^{24}$ sous la République. Certes, les textes juridiques qui définissent formellement les peines réservées au desertor et au transfuga ${ }^{25}$ datent de l'époque impériale, toutefois, de nombreux témoignages d'auteurs anciens permettent de connaître les châtiments réservés au déserteur sous la République. Denys d'Halicarnasse explique clairement que:

la mort est le châtiment traditionnel chez les Romains pour ceux qui ont abandonné leurs rangs ou quitté leurs enseignes. ${ }^{26}$

Tite-Live abonde dans le même sens en rappelant qu'“abandonner son poste, chez les Romains, est un crime puni de mort, et des pères ont sanctionné cette faute en faisant exécuter même leurs propres enfants". ${ }^{27}$ Plutarque raconte que, pendant la seconde guerre punique, le consul Marcellus, après avoir pris d'assaut la Sicile, n'a fait aucun mal aux habitants mais a mis à mort les déserteurs qui s'y étaient refugiés après les avoir battus de verges. ${ }^{28} \mathrm{Au}$ cours de ce même conflit, le consul Fabius a renvoyé les transfuges retrouvés à Rome où ils ont été tous battus de verges sur le Comitium avant d'être précipités du haut de la roche tarpéienne. ${ }^{29}$ Quant à Scipion, le second Africain, il fait preuve d'autant de fermeté à l'égard des déserteurs de son camp. En effet, "après la destruction de l'empire carthaginois", il fait subir aux transfuges des armées romaines les pires châtiments, et les "expose aux bêtes dans les spectacles qu'il donne au peuple". ${ }^{30}$ Paul Émile adopte une attitude tout aussi inflexible lorsqu'il a fait

écraser sous les pieds des éléphants les soldats étrangers coupables du même crime de désertion après la défaite du roi Persée. ${ }^{31}$

24 En Grèce, le déserteur, appelé aussi "le trembleur à la guerre", n'encourait pas la peine de mort mais, frappé d'atimie, il était exclu de la vie civique et subissait toutes sortes d'humiliations qui le conduisaient parfois au suicide (VeLHo 2003, 252-254).

25 Supra n. 2, 3, 4.

26 Dionys. Hal. IX,50.

27 Praesidio decedere apud Romanos capital esse et nec liberorum etiam suorum eram parentes sanxisse (Liv. XXIV,37,9). "La désertion était autrefois impardonnable", note Appien, $B C, \mathrm{~V}, 17$.

28 Plut., Marc., 17. Le même général fait fouetter à mort les déserteurs retrouvés après la prise de Léontinoï, Liv. XXIV,29.

29 Liv. XXIV,20. Tite-Live cite également l'exemple de C. Matiénus qui, accusé d'avoir déserté l'armée en Espagne, est battu de verges puis vendu à vil prix (Liv., Per., LV).

30 Val. Max. II,7,13-14. Tite-Live écrivait déjà qu'“à l'exemple de son frère naturel Paul-Émile, le vainqueur de la Macédoine, Scipion, donna des jeux publics, et exposa aux bêtes les transfuges et les fugitives" (Per., LI). Ces transfuges étaient destinés à périr sous la dent des bêtes féroces. Si cette forme de châtiment était assez rarement pratiquée sous la République (GARNSEY 1970, 129), elle figure officiellement parmi les peines réservées aux transfuges sous l'Empire (Dig. XLIX,16). Sur le sens du rôle des transfuges étrangers cf. Clavel-LÉvêQue 1984, 78-79.

31 Val. Max. II, 7,11 
Avant même l'ouverture de sa campagne de Contrébia, en 143, Q. Metellus ne fait-il pas clairement comprendre à ses hommes que, "celui qui prendrait la fuite et reviendrait au camp serait traité comme un ennemi et exécuté"? ${ }^{32}$

Notre intention n'est pas d'établir l'authenticité de telles sanctions encore moins leur efficacité dans la prévention de la désertion mais plutôt de mettre en évidence, à travers leur rigueur, la gravité de ce délit. Certes, les différents témoignages cités visent à magnifier les hautes qualités morales et militaires des Anciens. ${ }^{33}$ Mais ils attestent également leur volonté farouche de sauvegarder les valeurs traditionnelles de l'armée romaine ${ }^{34}$ en utilisant des moyens dissuasifs: le soldat romain doit vaincre ou mourir et ne jamais s'enfuir. Avant tout il fallait, pour refouler toute envie de déserter chez le soldat, remplacer la peur d'être blessé ou tué sur le champ de bataille par la crainte des humiliations et des peines encourues. Ainsi, en plus de la mort, ordinairement réservée au déserteur, le transfuge subit des châtiments déshonorants. Après la soumission de Carthage, Scipion le premier Africain

fait clouer les transfuges romains retrouvés sur la croix, comme déserteurs de la patrie, et fait périr les Latins sous la hache comme de perfides alliés. ${ }^{35}$

La gravité de ce crime qui relève de la trahison dans la mesure où il remet en cause et fragilise l'unité du nomen Romanum et de sa puissance justifie l'application des peines aussi infâmantes que la livraison aux bêtes ou la croix, ${ }^{36}$ châtiment juridiquement et traditionnellement réservé aux esclaves. Considérée dans la société romaine comme un supplice déshonorant, la croix est un servile supplicium. ${ }^{37}$ Pourtant, le général romain fait subir cette torture dégradante à ses milites, des citoyens romains. Choqué, mais n'osant pas critiquer un exemplum de la virtus républicaine, ${ }^{38}$ ValèreMaxime souligne cependant que ce type de châtiment, "si mérité soit-il" ne convenait pas à des "hommes de sang romain". En vérité, en commettant le délit de transfugium, le déserteur devient un proditor et un hostis, il perd automatiquement son statut de soldat et de citoyen. Par conséquent, il reçoit non pas des peines militaires, ${ }^{39}$ réser-

32 Val. Max. II,7,10. En 145, Q. Fabius Maximus fait couper les mains à tous les transfuges qui avaient fui les garnisons romaines et avaient été repris. “Ainsi les mains qui s'étaient rebellées et qu'on avait séparées des corps qui les portaient quand elles furent dispersées sur le sol qu'elles ensanglantaient, furent pour tous les autres la preuve tangible qu'il ne fallait pas oser commettre le même forfait", ajoute Valère-Maxime (II,7,11). De son côté, Appien ( $B C$, III,53), signale la pratique qui consistait à appliquer la decimatio lorsqu'un soldat abandonnait sa garde ou son poste de combat.

33 Phang 2008, 121-122; WolfF 2009, 106.

34 Val. Max. II,7,14.

35 Val. Max. II,7,12; Liv. XXX,43.

36 Et dans une moindre mesure le fouet qu'il faut ranger dans la panoplie des instruments de torture domestique réservés aux fils et aux esclaves, CANTARELla 2000, 139.

37 Le caractère infâmant de ce type de châtiment est souligné avec beaucoup d'éloquence par Cicéron, Rab. perd., V,16. La crucifixion est une peine de mort discriminatoire à Rome (CANTARELLA, 2000, 181-187).

38 Il dit lui-même que le premier Africain est un homme qui déteste la cruauté, un exemple de bonté (Val. Max. II, 7,12).

39 Parmi elles, on peut citer l'amende, le service extraordinaire, la dégradation, le congé infâmant. Théoriquement, les militaires ne sont condamnés ni aux mines ni mis à la torture (Dig., XLIX,16,3,1). 
vées aux soldats en faute, mais des châtiments dégradants. ${ }^{40}$ Par son comportement indigne d'un citoyen romain, le transfuge, en mettant en danger mortel son unité et l'Urbs, s'exclue lui-même de la cité.

\section{Fréquence et impunité du délit du desertio et de transfugium}

Infraction militaire très grave, la désertion se produit régulièrement pendant les guerres civiles..$^{41}$ Ces périodes de troubles la favorisent d'autant plus que, le soldat, ignorant de quel côté va aller le pouvoir, passe d'un chef à un autre. Ce constat se fait aussi bien au cours de la confrontation entre Marius et Sylla que pendant la guerre entre Antoine et Octave en passant par celle qui opposa César à Pompée. Individuellement ou massivement, les soldats abandonnent leur rang et rejoignent délibérément le camp ennemi parfois même en plein combat.

La disparition du général, mort ou en fuite se trouve quelquefois à l'origine de la désertion en groupes de milites. Ainsi, laissés à eux-mêmes, ils se tournent alors vers l'ennemi. ${ }^{42}$ En 49 av. J-C, à Corfinium, les hommes de L. Domitius Ahénobarbus se joignent massivement à César après la fuite de leur général ${ }^{43} \mathrm{D}$ 'autres fois, devant l'imminence de la défaite de leur camp ou bien attirés par les promesses plus alléchantes du camp adverse, les soldats passent à l'ennemi. C'est ainsi qu'en 83, pendant la campagne péninsulaire, Scipion et son fils Lucius sont victimes de la désertion massive de leurs hommes. Ils se retrouvent alors seuls et prisonniers de Sylla. ${ }^{44}$ Après la défaite du marianiste Norbanus à Faventia, 6000 soldats passent dans le camp de Metellus suivis d'une légion de Lucaniens. ${ }^{45}$ En Asie, C. Flavius Fimbria est abandonné par ses troupes qui s'unissent à Sylla condamnant ainsi leur chef à se donner la mort. ${ }^{46}$ En 82 aussi, à Préneste, les troupes de Marius, cinq cohortes d'infanterie et deux de cavalerie, jettent leurs enseignes et se tournent vers Sylla. ${ }^{47}$ Pendant les dissensions civiles qui ont suivi l'assassinat de César, les vagues de désertions se révèlent aussi nombreuses. En novembre 44, au moment où Antoine s'apprête à se rendre au Sénat, il apprend que deux de ses

40 Mais, sous l'Empire, si les peines réservées aux déserteurs s'allègent, tel n'est pas le cas des transfuges (Dig., XLIX,16,9). Après avoir été dégradé, le transfuge était mis à la torture avant de subir la peine capitale (Dig., XLIX,16,3 et XLVIII,1,3,6). Dans le code militaire français, le déserteur en temps de paix comme en temps de guerre n'encourt pas la peine de mort mais plutôt l'emprisonnement allant de cinq à vingt ans (Code, Livre III, Titre II, article 413). En revanche, la trahison et l'espionnage sont punis de la réclusion criminelle à perpétuité et de 750000 euros d'amende (Code, articles 476-1 et 476-5).

41 Tacite (Hist., I,51,3) explique que "la discipline, inexorable en temps de paix, se relâche à l'occasion des guerres civiles".

42 Sur les raisons psychologiques qui poussent les soldats romains à la désertion, WoLfF 2009, 264-266.

43 Le pompéien qui échoue dans sa tentative de bloquer la route à son ennemi est livré par ses hommes à César (Caes., $B C$, I,23).

44 App., $B C, \mathrm{I}, 10,85$.

45 App., $B C, \mathrm{I}, 10,91$.

46 Plutarque ajoute même qu'ils embrassèrent les hommes de Sylla et les aidèrent dans leurs travaux (Plut., Sull., 25).

47 App., $B C$, I, 10,87; I, 10,90. 
légions se sont ralliées à Octave qui leur a offert 500 drachmes à chacun. ${ }^{48}$ Enfermé dans Pérouse, certains hommes de L. Antonius s'échappent pour retrouver Octave. ${ }^{49}$ Aucune de ces désertions n'a été suivie d'une dénonciation ni d'une sanction pénale. ${ }^{50}$ Du moins, ni Appien, ni César ne le signalent. En 49, Caton s'oppose énergiquement au fils aîné de Pompée qui avait décidé de sévir contre les déserteurs de Pharsale. ${ }^{51}$

Ces nombreux mouvements de désertion dans l'armée font croire qu'abandonner son poste ne constitue pas un délit grave tant du côté des soldats, qui changent souvent de camp, que de celui de leurs chefs, qui ripostent par des sanctions molles si ce n'est par une attitude "conciliante". Pourtant, cette mobilité des soldats remet fondamentalement en cause la valeur du sacramentum qui confère au général le droit de vie et de mort sur ses hommes qui doivent lui "obéir et exécuter ses ordres de toutes leurs forces". ${ }^{52}$ Avant d'accepter les transfuges dans leurs troupes, les commandants en chef exigent qu'ils leur prêtent serment, ${ }^{53}$ lequel, malgré les dysfonctionnements des armées civiles, reste le fondement de l'armée romaine. Mais ces serments personnels, une arme qu'utilisent les généraux pour attacher les soldats à leur cause ${ }^{54}$ ou pour les rappeler à l'ordre ne les empêchaient pas de déserter. Finalement, à force de passer d'une armée à une autre et à force de prêter serment à différents chefs militaires, le transfuge des guerres civiles ne donne pas l'impression de passer à l'ennemi en changeant de camp mais seulement de changer de commandant. Pourtant, cette mobilité souvent accompagnée d'un nouveau serment, pose un problème juridique réel. Si d'un côté le second serment peut rendre caduc le premier à cause du changement d'identité du général, ${ }^{55}$ de l'autre, en revanche, le premier serment garde une valeur plus grande compte tenu de son antériorité. Á Utique le pompéien Sextus Quintilius Varus n'a pas manqué de faire remarquer aux soldats de Curion qu'ils étaient, avant de s'unir à César ${ }^{56}$ des hommes de Pompée, qu'ils ont d'abord prêté serment à L. Domitius et à lui-même son questeur et que, par conséquent, ils ne peuvent ni ne doivent porter les armes contre eux. Mais ces soldats doivent-ils fidélité à César ou à Varus? Le second serment prêté à César peut-il annuler les effets du premier? Ce passage du Bellum civile, qui livre plutôt les impressions de César, ${ }^{57}$ partie prenante dans ce conflit, reste muet sur l'opinion des soldats. Mais ici la caducité du premier serment qui justifie l'attitude des soldats s'explique par des raisons

48 Il leur promit 5000 drachmes à chacun si, en cas de guerre, ils étaient victorieux (App., $B C$, III,45, 185; III,47,191). Il passait tous les jours dans le camp de César un grand nombre de déserteurs, et l'on en dénonçait encore beaucoup d'autres, comme soupçonnés de vouloir suivre leur exemple (Plut., Brut., 39).

49 App., $B C, \mathrm{~V}, 38$. Après Naulocques, les hommes de Lépide passent du côté d'Octave (App., $B C, \mathrm{~V}, 125-126)$.

50 Seul le roi de Numidie, allié de Pompée, s'est montré inflexible envers les déserteurs numides en 46. Juba fait crucifier tous ceux qui avaient abandonné leur poste pour rejoindre César (Ps. Caes., BA, 66,4).

51 Plut., Cat. min., 55.

52 Plb. VI,21,2. Sur le contenu du sacramentum, cf. Dionys. Hal. XI,43,2 et Hinard 1993, 252-263.

53 App., $B C, \mathrm{IV}, 8,62$; Caes., $B C, \mathrm{I}, 23,5$.

54 "In the late Republic, the military oath became more and more a personal bond to the individual commander, first imposed by Sylla on his troops" (Phang 2008, 119).

55 WolfF 2009, 331-332.

56 Juste après leur prestation de serment à Corfinium, les hommes de L. Domitius ont été dirigés vers la Sicile (Caes., $B C$, I,23; I,25).

57 Dans son Introduction, P. Fabre rappelle que même si les faits relatés dans La guerre civile sont exacts, César les colore habilement car elle "doit avant tout être considérée comme une apologie" justifiant toutes ses décisions et dépréciant les actions de ses adversaires (voir FABRE, trad., 1965, 28-29). 
militaires. En fait, en abandonnant ses troupes au milieu du danger pour prendre la fuite et en se retrouvant prisonnier de César, le proconsul L. Domitius Ahénobarbus perd son imperium ce qui libère du coup ses soldats de leur serment. La multiplication des serments personnels affaiblit le sacramentum et encourage inévitablement la désertion.

À la place d'une condamnation, déserteurs et transfuges sont plutôt accueillis avec indulgence (sentiment parfois voilé d'une légère teinte de méfiance attestée par l'exigence d'un serment d'obéissance) et empressement aussi bien du côté du camp adverse que de celui du camp d'origine. S'ils sont dépités par les désertions qui se produisent dans leur camp, les chefs militaires se montrent toujours très satisfaits de recueillir les transfuges. En janvier 49, César accepte les hommes de L. Domitius Ahénobarbus à qui il demande d'abord de lui jurer fidélité. ${ }^{58}$ En Afrique, même si César prend la précaution de mettre à l'écart les transfuges gétules, il les accepte après les avoir couvert d'argent et d'éloges. ${ }^{59}$ Comment des commandants de l'armée qui se servent des transfuges pour renforcer de manière conséquente leurs effectifs et afficher leur force peuvent-ils les sanctionner? L'arrivée massive des soldats du camp opposé est utilisée par les responsables militaires comme une preuve éloquente de leur position favorable, présage d'une proche victoire, et un moyen efficace pour effectuer une ponction dans les forces de l'ennemi. En affichant la supériorité numérique de leurs troupes, ils cherchent simultanément à intimider l'adversaire tout en appâtant ses soldats mais aussi à rassurer leurs hommes afin de les fidéliser. En Espagne, le légat de Pompée, M. Terentius Varron, a recouru à cet argument numérique pour galvaniser et fidéliser ses troupes. Sans se soucier de la véracité de ses propos, il clame Caesarem proelia fecisse, magnum numerum ab eo militum ad Afranium perfugisse ${ }^{60}$ En mai 48, Pompée est animé par le même esprit lorsqu'il fait promener dans tous ses postes les deux transfuges allobroges qui ont déserté le camp de César. Ce dernier, de son côté aussi, se glorifie de recevoir les transfuges qui proviennent des quartiers de Pompée. Il prétend même que, jusqu'à la désertion des deux Allobroges,

personne, ni fantassin, ni cavalier, n'était passé de César à Pompée, tandis que, chaque jour, des déserteurs pompéiens gagnaient le camp de César. ${ }^{61}$

En Afrique également, César recueille les Gétules et les Numides qui ont déserté en foule le camp de Scipion et de Juba et leur assure même une solde. ${ }^{62}$ En 46, de nombreux soldats des quatrième et sixième légions de Scipion passent dans le camp de César et 1000 cavaliers gétules quittent la garde royale numide pour rejoindre César à

58 Caes., $B C$, I,23. Plutarque ne mentionne pas le serment mais indique que les soldats se joignent à lui (Plut., Caes., 35,1).

59 Ps. Caes., $B A, 35,5$.

60 "César avait subi des défaites et qu'un grand nombre de ses soldats avaient déserté pour passer au camp d'Afranius" (Caes., BC, II,18).

61 nemo aut miles aut eques a Caesare ad Pompeium transierat, cum paene cotidie a Pompeio ad Caesarem perfugerent (Caes., $B C$, III,61).

62 Ps. Caes., $B A, 32,1-4 ; 35,5$. Il explique qu'il crée dans son camp un quartier pour ces transfuges. Les généraux encouragent en fait la désertion (même si les enjeux diffèrent) au lieu de se comporter comme le consul Fabricius pendant la guerre contre Pyrrhus. Tite-Live raconte que le consul, au lieu d'utiliser les services d'un déserteur qui est passé dans son camp et qui lui promettait d'empoisonner le roi, il le renvoie auprès de Pyrrhus (Liv., Per., XIII.). 
Uzitta. $^{63}$

Dans le camp d'origine également, le retour du déserteur ou du transfuge est non seulement accepté mais aussi recherché. Au lieu de sanctionner transfuges et déserteurs, les chefs des armées s'évertuaient plutôt à les récupérer. Antoine s'abaisse jusqu'à se rendre à Alba Fucens pour tenter de reconquérir les transfuges de la "Quatrième" légion et de la "Martialis" qui l'accueillent à coups de flèches. ${ }^{64}$ L'exemple du lieutenant de Sextus Pompée, Ménodorus, montre bien avec quelle légèreté les commandants en chef traitent les transfuges de leur propre armée. Après sa dispute avec Sextus, Ménodorus quitte son armée et rejoint Octave avec ses navires. Déçu par les promesses de ce dernier, il fait volte-face et retourne auprès de Sextus Pompée qui lui accorde tout naturellement son pardon. ${ }^{65}$ Mais il rejoint, une nouvelle fois, Octave qui, soucieux de rassembler le maximum d'hommes autour de lui en vue d'une victoire militaire et politique, l'incorpore dans son armée. ${ }^{66}$ De peur de tarir une source de recrutement facile et accessible au profit de l'adversaire, les chefs militaires restent souples vis-à-vis des transfuges qui sont en position de force.

\section{La desertion utilisée comme une arme militaire et politique}

L'altération des liens fondés sur le sacramentum entre le général et ses hommes instaure de nouveaux rapports qui, désormais reposent sur l'intérêt au détriment de la fidélité et de l'obéissance. Ce bouleversement des fondements de l'armée favorise un relâchement disciplinaire qui profite aussi bien aux milites qu'à leurs responsables. Conscients de la nécessité de disposer d'une bonne armée qui seule peut les conduire à assouvir leurs ambitions politiques, ${ }^{67}$ les chefs militaires tolèrent les excès et écarts de conduite de leurs hommes de peur de les perdre au profit du parti opposé. ${ }^{68}$ De leur côté, les soldats, préoccupés par leur misère, cherchent aussi à s'enrichir en se vendant aux plus offrants. ${ }^{69}$

Les commandants en chef doivent s'acquitter désormais d'une triple tâche souvent contraire aux principes fondateurs de l'armée: garder leurs hommes, reconquérir les déserteurs de leur propre camp et récupérer les transfuges de l'adversaire. Ainsi, tantôt ils luttent contre la désertion dans leur propre camp, tantôt ils l'encouragent dans

\footnotetext{
63 Ps. Caes., $B A, 56,3$.

64 App., $B C$, III,45.

65 App., $B C, \mathrm{~V}, 80 ; \mathrm{V}, 96$.

66 App., $B C, \mathrm{~V}, 100-102$.

67 Appien dénonce avec insistance cette dégradation des mœurs et règles militaires pendant les guerres civiles (App., BC, V,17; JAL 1962, 7-8). Nous ne reviendrons pas sur les levées massives et irrégulières d'armées pendant les guerres civiles, sujet largement traité par HARMAND (1967, 246-256) et CoSmE (2007, 53-55).

68 Appien explique que les généraux n'osaient pas toucher leurs soldats de peur d'exciter leur rage (App., $B C, \mathrm{IV}, 35,14)$.

69 "Le soldat des guerres civiles" attend de son général qu'il l'enrichisse. Il est d'ailleurs largement soutenu par les chefs militaires qui achètent ouvertement la fidélité de leurs hommes. La remarque de Tacite selon laquelle "l'argent est le nerf de la guerre civile" revêt toute sa pertinence, (Tac., Hist., II,84,1). HARMAND souligne que les nombreuses désertions pendant les guerres pontiques découlent de la misère du soldat romain (HARMAND 1967, 286-288).
} 
le camp opposé. De fait, l'arrivée en grand nombre de déserteurs ou de transfuges ne peut que renforcer les rangs de celui qui les accueille. Elle reste même parfois déterminante pour l'issue des affrontements. En janvier 49, César exploite habilement les désertions massives des troupes pompéiennes de Corfinium et de Brindes pour grossir ses effectifs. Subséquemment, il dirige les transfuges de Corfinium vers la Sicile auprès du propréteur $\mathrm{C}$. Curion qui a pu, de la sorte, disposer de trois légions avant de s'embarquer pour l'Afrique. ${ }^{70}$ César enrôle automatiquement les neuf cohortes pompéiennes qui ont abandonné leurs chefs à Brindes. ${ }^{71}$ En 44, c'est également grâce aux deux légions d'Antoine, la "Quatrième" et la "Martialis", que "le jeune Octave", qui ne possédait auparavant qu'une légion de nouvelles recrues et deux légions de vétérans, a pu se retrouver à la tête d'une véritable armée. ${ }^{72} \mathrm{C}$ 'est avec cette dernière, ${ }^{73}$ et seulement en qualité de propréteur, qu'il a contribué à la victoire des armées sénatoriales à Modène. ${ }^{74}$

En somme, aux yeux de l'ennemi, les transfuges se révèlent utiles quand, par leur nombre, ils renforcent leurs troupes mais aussi lorsque, par les informations "fraîches" et souvent authentiques qu'ils livrent "in order to establish their credentials more quickly" 75 ils permettent d'atteindre l'adversaire. Membre à part entière de leur unité d'incorporation et occupant même quelquefois des postes stratégiques, ${ }^{76}$ lorsqu'il passe à l'ennemi, le transfuge devient une mine d'informations qui se révèlent quelques fois cruciales. ${ }^{77}$ Dans La guerre civile, César évoque souvent les transfuges qui, sous sa plume, se transforment en de véritables agents de renseignements. ${ }^{78}$ D'ailleurs, il avoue avoir sollicité à maintes reprises leurs services dévoilant par la même occasion le rôle stratégique et parfois décisif des transfuges sur l'échiquier de la guerre. En Afrique, c'est grâce aux transfuges gétules, venus le rejoindre en grand nombre, qu'il put échapper aux pièges tendus par les Pompéiens. C'est également par l'intermédiaire des déserteurs numides, venus dans son

70 Caes., BC, I,25; I,31; II,32.

71 Il s'agit des 6 cohortes du préteur L. Manlius et des trois cohortes de Rutilius L. Terracine (Caes., BC, I,24).

72 App., $B C$, III,47,191.

73 Le consul Hirtius avoue que les légions d'Antoine qui ont déserté constituent "l'élément le plus fiable" de leur armée compte-tenu de leur expérience et de leur qualité physique (App., $B C$, III,65,266).

74 App., BC, III, 69-79. Les batailles de Forum Gallorum et de Modène sont certes une confrontation entre les armées sénatoriales, sous la conduite des consuls Pansa et Hirtius, et les troupes d'Antoine mais elles sont surtout un affrontement entre les soldats d'Antoine, c'est-à-dire ceux des légions qui ont déserté et ceux des légions qui sont restées. De fait, Modène a mis en jeu l'honneur de chacune des légions d'Antoine, les unes et les autres se reprochant leur lâcheté ou leur trahison.

75 Austin - RANKov 1995, 74-75; SHELdON 2009.

76 Appien relève clairement que, même des hommes illustres, appâtés par la fortune ou la gloire, devenaient des transfuges (App., $B C, \mathrm{~V}, 17$ ).

77 En effet, les déserteurs et les transfuges "provide information of a type which cannot be identified from the roman agencies alone, which in their turn cannot penetrate every area where military activity might be taking place, nor reach every place where a strike can with advantage be undertaken" (AUSTIN - RANKOV 1995, 75).

78 La même remarque est valable aussi dans la Guerre d'Afrique. On peut constater, ici, que César n'a pas seulement utilisé les prisonniers de guerre pour connaître les desseins de l'ennemi, il a recouru à multiples reprises aux services des déserteurs aussi bien pendant la guerre civile que durant ses campagnes en Gaule (Caes., $B G$, III,18; V,18). 
camp, après la bataille de Ruspina, que César a su que l'ennemi envisageait d'user de la même stratégie que celle déjà expérimentée, avec succès du reste, contre Curion à Bagradas. ${ }^{79}$ En effet, ils révèlent à César les desseins de T. Labiénus ${ }^{80}$ qui, caché en embuscade, comptait le prendre par surprise avec ses troupes. Averti, César laisse alors passer plusieurs jours avant de reprendre sa marche ce qui finit par démoraliser et lasser T. Labiénus et ses hommes. ${ }^{81}$ Des renseignements provenant de transfuges lui ont aussi évité le piège de Vaga en mars 46. En effet, au moment où la garnison césarienne devait se rendre à Vaga, pour recevoir des fournitures pour la campagne, Juba fait encercler la place. Averti par un transfuge numide, César annule alors 1'opération. ${ }^{82}$

Les adversaires de César ont également employé les mêmes stratagèmes. Dans ses quartiers de Dyrrachium, Cn. Pompée affiche toute sa fierté en recevant les deux cavaliers Allobroges, Ecus et Roucillus, qui ont abandonné leurs enseignes. À en croire César, le rôle de ces deux transfuges a été décisif dans l'issue de l'affrontement de sa flotte contre celle de Pompée à Oricum en 48. Dépité, il avoue que la trahison des deux frères allobroges lui a valu un cruel échec parce qu' "ils connaissaient tous les détails de son camp". ${ }^{83}$ Lorsque les allobroges ont rejoint le camp de Pompée, ils le mirent au courant des moindres faiblesses des travaux de retranchement entrepris par les troupes de César. Et, cette information s'est révélée plutôt capitale. C'est ainsi que Pompée et ses hommes ont réussi à surprendre les cohortes de la neuvième légion conduites par le questeur Lentulus Marcellinus. En effet, lorsqu'inopinément les Pompéiens surgissent de leurs chaloupes et petites embarcations à la pointe du jour, ils trouvent les Césariens pratiquement à découvert et les assaillent de projectiles, car les travaux d'élévation du double rempart de protection étaient inachevés. En insistant sur la trahison des deux allobroges, César cherche d'une part à déprécier la valeur militaire de son adversaire qui a bénéficié du soutien de transfuges, d'autre part à justifier sa défaite. ${ }^{84}$ En réalité, son échec est en partie imputable à son imprudence car il a pris le risque d'entreprendre des travaux considérables en peu de temps.

Cependant, l'arme stratégique que constitue l'exploitation des renseignements livrés par le transfuge est à double tranchant. Curion en a fait une expérience funeste à Utique, en juillet $49 .{ }^{85} \mathrm{Il}$ apprend par les transfuges que Juba, occupé à étouffer les soulèvements des habitants de Leptis, charge Saburra, son lieutenant, de conduire l'armée numide. Ceci sous-entend, bien sûr, que les troupes de Saburra sont peu considérables puisqu'une bonne partie des soldats devait rester avec Juba. ${ }^{86}$ Alors,

79 Ps. Caes., BA, 19, 1-2. Armé de ces renseignements stratégiques, César s'est mis à fortifier son camp et à renforcer ses troupes pour faire face aux effectifs très élevés des Pompéiens.

80 T. Labiénus est un ex-lieutenant de César et également un transfuge (Caes., $B C$, I, 15,2; III,13,3).

81 Ps. Caes., $B A, 66,1-3$. T. Labiénus est finalement mis en déroute par les troupes de César.

82 Ps. Caes., $B A, 74,1-2$. César considère ce renseignement comme un signe de "la bienveillance et de la faveur des dieux" à son égard.

83 Caes., BC, III,61. Rappelons que les cavaliers Ecus et Roucillus ont été aux services de César durant toutes ses campagnes en Gaules (Caes., BC, III,59-60). Plutarque raconte aussi que Brutus fut profondément affecté par la désertion d'un de ses meilleurs officiers, Camulatus, qui, en plein combat, rejoint les rangs ennemis (Plut., Brut., 49).

84 Sans insister sur les risques qu'il a pris en entreprenant de tels travaux en un temps aussi court.

85 Caes., $B C$, II,42-43.

86 Caes., BC, II,37; II,39. 
rassuré par les renseignements fournis par les transfuges et corroborés par les prisonniers de guerre, Curion, voulant profiter de cette "aubaine", attaque avec précipitation l'ennemi à Bagradas. En réalité, le roi numide était convenu avec Saburra de le suivre à distance et de le rejoindre une fois que la bataille éclate. Juba et son lieutenant ont-ils fait circuler sciemment ces fausses informations dans leur camp? Ou bien les transfuges et les prisonniers n'ont rapporté que les faits c'est-à-dire qu'ils n'ont vu que Saburra à la tête de l'armée? Dans tous les cas, César qui cherche, comme à Oricum, à justifier la défaite de son infortuné lieutenant à Bagradas, ne donne aucune explication quant à la manière dont ces renseignements inexacts ont été distillés.

À sa qualité d'informateur, le transfuge peut adjoindre celle d'espion et de propagandiste. Ce rôle se révèle essentiel dans ce "conflit moral et psychologique" qu'est la guerre civile où il faut convaincre de la légitimité et de la justesse de sa cause. ${ }^{87}$ Qui mieux que le transfuge, compte tenu de sa connaissance des deux armées, est assez bien placé pour jouer ce rôle? Le cours des guerres entre citoyens romains est souvent rythmé par des intrusions assez récurrentes dans les quartiers de l'adversaire faisant ressembler aux deux camps à des vases communicants. La perméabilité des lignes ennemies reste déconcertante. Elle s'explique en partie par la sympathie et la fraternité qui lient parfois les soldats car, avec la fréquence des désertions, des milites qui ont auparavant partagé la même unité se retrouvent dans des camps opposés. À juste titre, P. Jal fait remarquer que

la guerre civile est l'âge d'or de l'espionnage. Jamais peut-être belligérant ne fut aussi bien renseigné sur les moindre faits et gestes de l'ennemi qu'il le fut en cette période $^{88}$

Naturellement, l'adversaire rentre chez l'ennemi pour débaucher ouvertement ou en cachette les soldats de l'adversaire en leur promettant argent et gratifications. Ce travail est facilité par l'état d'esprit du soldat qui ne se joignait pas à un tel ou tel autre chef par conviction ou par fidélité ou, du moins, par respect des principes de l'armée mais tout simplement parce que l'un ou l'autre lui offrait plus. ${ }^{89}$ En Afrique, les transfuges gétules, récompensés par les bienfaits de César, deviennent de véritables agents de propagande auprès de leurs compatriotes. C'est en partie grâce à eux qu'il a bénéficié du soutien massif des Numides et des Gétules qui ont retourné leurs armes contre les Pompéiens..$^{90}$ Toutefois, l'auteur de la guerre d'Afrique, préoccupé de dépeindre un César plutôt clément et désintéressé, n’insiste pas sur cette propagande dans laquelle César tient un rôle actif à travers les promesses tentantes faites aux populations autochtones. ${ }^{91}$

$87 \mathrm{Jal}$ aborde les questions relatives aux enjeux et pratiques de la propagande en période de guerre civile (JAL 1963, 83-230).

88 JAL $1963,345$.

89 Sur l'état mental et psychologique du soldat des guerres civiles, JAL 1962, 11-15.

90 Ps. Caes., BA, 55. Certains se rallient à sa cause en souvenir de Marius (Ps. Caes., BA, 32,3).

91 C'est plutôt Dion Cassius qui, connaissant le fin stratège et diplomate qu'est César, révèle qu'il faisait distribuer des tracts dans lesquels il garantissait la possession de leurs terres aux Numides et promettait l'amnistie aux citoyens romains (D.C. XLIII,5,1-4). 
L'utilité des transfuges dans l'issue des conflits ne fait aucun doute et explique, dans une certaine mesure, l'attitude conciliante des chefs militaires. Ces mêmes responsables, directement ou indirectement, incitent à la trahison les soldats de l'ennemi. Octave en est le prototype parfait. ${ }^{92} \mathrm{En} 44$, il exploite le mécontentement de l'armée de Brindes qui nourrissait deux griefs à l'encontre d'Antoine: l'impunité de l'assassinat de César et l'injustice de l'application de la decimatio. Conscient de l'état d'esprit des troupes de son adversaire, il envoie ses agents de propagande auprès d'elles. ${ }^{93} \mathrm{Le}$ choix de ce moment stratégique lui a valu le ralliement des deux légions d'Antoine, en novembre 44. Octave va plus loin encore avec les soldats de Lépide après Naulocques. Il pénètre en personne dans le camp de son ennemi et tente de débaucher ouvertement les soldats mécontents ${ }^{94}$ et démoralisés par cette guerre éprouvante qui se prolongeait. ${ }^{95}$ Cette violation flagrante et répétée des règles de la guerre semble même être cautionnée par le Sénat, qui ne réagit pas aux dénonciations d'Antoine condamnant les manœuvres déloyales de son adversaire. ${ }^{96}$ Mais, largement dominé par les partisans de Cicéron qui comptaient plutôt sur Octave pour abattre Antoine, le Sénat ne réagit point.

Depuis Rome précisément au Sénat, les hommes politiques entretiennent l'indiscipline militaire en développant l'hostilité des soldats envers leurs généraux pour atteindre l'ennemi aux dépens même des règles fondatrices et directrices de l'armée. Un cas édifiant est, ici, l'exemple de l'opposition entre le sénateur Cicéron et le consul Antoine. Cicéron accuse son adversaire, qui, du reste, a ruiné ses projets d'amnistie, ${ }^{97}$ de poursuivre les mêmes ambitions que César: l'instauration du pouvoir personnel. ${ }^{98}$ En octobre 44, à Brindes, les soldats d'Antoine refusent non seulement d'acclamer leur général à qui ils reprochent de ne pas venger le meurtre de César, mais aussi quittent l'assemblée sans permission après l'avoir tourné en ridicule. Excédé par l'insolence de ses hommes, Antoine décide de sévir collectivement en appliquant la decimatio. ${ }^{99}$ Alors, successivement, les 22 et 28 novembre, la "Légion de Mars" et la "Quatrième Légion" abandonnent le "parti" d'Antoine, en violation des usages militaires dans un contexte où tous les référents traditionnels des codes politiques sont bouleversés, pour rejoindre Octave. ${ }^{100}$ Devant le Sénat, en janvier 43, Cicéron

92 Sylla a recouru de façon récurrente à la corruption pour gagner les troupes ennemies à sa cause et pour fidéliser ses hommes. Cette attitude est fortement dénoncée par Plutarque (Plut., Sull., 12,9).

93 Ils étaient chargés de distribuer un "grand nombre de libelles" aux soldats d'Antoine, de flétrir l'avarice et la cruauté de leur général et de leur promettre cadeaux et privilèges (App., $B C$, III,44). Octave s'attachait ces hommes au moyen de cadeaux.

94 Ses soldats lui reprochaient d'avoir laissé une part du butin à l'ennemi.

95 App., $B C, \mathrm{~V}, 124-125$.

96 App., $B C$, III,45.

97 Après avoir fait semblant de souscrire à une amnistie pour l'attentat contre César, idée fortement défendue par Cicéron qui voulait un retour à la concorde, Antoine a rallumé la fureur du peuple contre les meurtriers en exposant publiquement les habits ensanglantés et portant les différents coups d'épée reçus. Cicéron ne lui a pas pardonné pas ce geste qui risque d'annihiler ses efforts (Plut., Cic., 42; Plut., Ant., 14).

98 Plut., Cic., 43.

99 La loi militaire prescrit d'exécuter un sur dix soldats en cas de désobéissance.

100 L'application de la decimatio n'est pas étrangère à cette désertion massive. Toutefois, les offres tentantes d'Octave ont fortement influencé les troupes d'Antoine car, au lieu de rejoindre les armées sénatoriales, qui représentent la République, elles se joignent à Octave. 
critique violemment son adversaire et lui reproche d'avoir pris une mesure disciplinaire abusive. L'argument avancé par le sénateur reste sans fondement juridique à partir du moment où l'exercice du commandement militaire inclut, dès les origines de Rome, le droit de punir. Or, Antoine, en dépit d'une application peu traditionnelle de la décimation, ${ }^{101}$ en sa qualité de consul, "had possessed imperium militiae over his soldiers, from which they were no appeal". ${ }^{102}$ Pison fait remarquer à Cicéron et à l'ensemble du Sénat que:

aucun commandant n'a eu à rendre compte de tels faits. Car les lois n'ont pas jugé qu'il serait de notre intérêt que le chef soit responsable devant ses soldats; et qu'il n'y a rien de pire non plus que l'indiscipline dans une armée..., et pas un parent des soldats en question n'en blâme Antoine, c'est Cicéron qui le fait. ${ }^{103}$

En fait, Antoine s'est servi de l'exécution de ces hommes pour, non seulement faire revenir la discipline dans les rangs, mais pour donner aussi l'exemple ce qui a plutôt produit l'effet inverse. Dans les faits, le consul n'a appliqué que partiellement la règle de la decimatio, ce qu'il a beaucoup regretté par la suite. ${ }^{104}$ Mais, pour des raisons évidentes, Cicéron ne le mentionne pas. Punition certes bien sévère pour quelques éclats de rire et railleries et exceptionnelle aussi en ces temps de guerre civile où les généraux ménagent avec beaucoup de délicatesse leur troupe ! Néanmoins, cette mesure disciplinaire se justifie par le climat particulier qui règne dans le camp d'Antoine investi presque en permanence par les espions d'Octave. Pourtant, Cicéron, emporté par sa haine et animé par ses ambitions politiques va jusqu'à s'attaquer à une tentative de redressement de l'armée. Après avoir critiqué un consul de la République en exercice, Cicéron, avec la complicité du Sénat, récompense les soldats de la "Légion de Mars" et ceux de la "Quatrième Légion" d'avoir commis le délit de transfugium en leur distribuant une somme d'argent prélevée dans le trésor public. ${ }^{105}$ Mieux, après Modène, le sénateur promet à ces mêmes déserteurs une prime de victoire de 5000 drachmes et leur accorde le droit permanent de porter une couronne de feuillage pendant les fêtes. Antoine mesure l'inconséquence de l'acte du sénateur lorsqu'il lui fait remarquer qu'en offrant

des primes aux légions qui ont déserté mais pas celles qui sont restées, il corrompt par là les mœurs militaires moins à son détriment qu'à celui de Rome. ${ }^{106}$

101 Cosme 1993, 67-68.

102 Phang 2008, 115.

103 App., $B C$, III, 8,56 .

104 Selon Appien, il ne fait exécuter qu'un petit nombre de soldats. Ni Crassus ni César, qui ont appliqué la decimatio à leurs hommes, n'ont eu à rendre compte au Sénat et n'ont reçu aucun blâme non plus (App., $B C, \mathrm{I}, 14,118 ; \mathrm{II}, 7,47)$.

105 App., $B C$, III, 10,303.

106 App., BC, III,8,232-233. 
Les agissements du sénateur, orientés par des ambitions politiques personnels, mettent en danger la Res publica ${ }^{107}$ et les transfuges en sont ici un signe plus que des acteurs. Pour des raisons politiques, Cicéron, qui cherchait à évincer Antoine et laisser la province de la Gaule Cisalpine à Décimus Brutus, a encouragé avec le Sénat la désertion en faisant intervenir les milites dans la vie politique. En réalité, ce qui intéresse Cicéron c'est moins le traitement infligé aux soldats que l'utilisation de cet événement pour faire déclarer Antoine ennemi public. ${ }^{108}$

En somme, les responsables de l'armée depuis les camps et les hommes politiques depuis le Forum, utilisaient les transfuges pour le triomphe de leur "parti" et encourageaient aussi l'indiscipline militaire au détriment des intérêts de l'Etat.

$\mathrm{Au}$ terme de cette étude, il nous reste à répondre à une dernière interrogation. $\mathrm{La}$ désertion est-elle ou peut-elle être un véritable crime en période de guerre civile? Entorse au règlement militaire passible de la peine de mort surtout lorsqu'elle s'accompagne de trahison, la désertion est condamnée à l'unanimité par les sources qui ne font, du reste, aucune distinction entre les périodes de guerre civile et de guerre étrangère. Toutefois, l'incitation à la désertion, son orchestration et sa récupération par les chefs de l'armée et des partis politiques, la mollesse des sanctions ou plutôt leur quasi-absence et l'achat de la fidélité des soldats nous poussent à croire que même si, dans le principe, abandonner son rang pour rejoindre l'adversaire reste sans aucun doute une violation de la loi militaire, dans le contexte particulier des luttes intestines, cet acte demeure un délit mineur et toléré voire encouragé. Il s'agit d'un outil stratégique qu'utilisent les hommes politiques à la fin de la République pour évincer leurs ennemis. Soldats comme chefs militaires des guerres civiles ne considèrent pas la désertion comme une faute grave. Chaque parti politique prétend défendre et sauvegarder les principes fondateurs de la Res publica et de la Patria et cette similitude dans les arguments politiques soutenus allège fortement le poids de la trahison. En dépit de l'atrocité affreuse des carnages qui caractérisent les conflits civils, les hommes de troupe n'ont pas en face d'eux de véritables hostes. Si la ligne de démarcation est bien nette entre armées étrangères en période de bellum externum, elle l'est beaucoup moins lorsqu'il s'agit d'une guerre civile.

\section{Bibliographie}

Austin, N. J. E. - Rankov, N. B. (1995): Exploratio: Military and political intelligence in the roman world from the Punic war to the battle of Andrianople, London.

Boulanger, A. (trad.), (1960): Cicéron. Pour Rabirius, Paris, Les Belles Lettres.

Bouvet, A. (TRAD.), (1997): Pseudo-César. Guerre d'Afrique, Paris, Les Belles Lettres.

107 Pison, s'adressant aux sénateurs, leur dit: "le degré d'indiscipline et d'arrogance qu'Antoine a trouvé dans son armée est bien illustré par la défection de deux légions auxquelles, vous, par décret aviez ordonné de servir Antoine, mais qui ont choisi de l'abandonner. ... puissiez-vous ne jamais regretter cet exemple" (App., $B C$, III, 8,232 ).

108 Non seulement Antoine s'est emparé de la Gaule sans le consentement du Sénat, qui lui avait attribué la Macédoine mais il a aussi transféré en Italie l'armée destinée à combattre les Parthes. 
Buonocore, R. A. (2002): "Relaciones, información, espionaje y servicios de inteligencia en Roma”, Semanas de Estudios Romanos XI, Valparaiso, 65-83.

Cantarella, E. (2000): Les peines de mort en Grèce et à Rome. Origines et fonctions des supplices capitaux dans l'Antiquité classique, Paris.

Chaumartin, F.-R. (Trad.), (2005): Sénèque. De la clémence, Paris, Les Belles Lettres.

Clavel-LévêQue, M. (1984): L'Empire en jeux. Espace symbolique et pratique sociale dans le monde romain, Paris.

Code $=$ Code de justice militaire, Éditions des journaux officiels, Paris, 2010.

Collins, J. (1972): “Caesar as Political Propagandist”, ANRW 1, 922-966.

Combès, R. (TRAD.), (1995): Valère-Maxime. Faits et dits mémorables, Paris, Les Belles Lettres, 1995.

Combes-Dounous, I. (TRAD.), (1993): Appien. Les guerres civiles à Rome, Paris, Les Belles Lettres, 1993.

Cosme, P.

(1993): "Le livret militaire du soldat romain", C.C.G.G. 4, 67-68.

(2003): "Le châtiment des déserteurs dans l'armée romaine", Revue Historique de Droit Français et Étranger 81, 287-307.

Daremberg, Ch. - Saglio, Ed. - Pottier, E. (1877-1919): Dictionnaire des Antiquités grecques et romaines, Paris.

FABre, P. (TRAD.), (1965): César. La guerre civile, Paris, Les Belles Lettres.

Flacelière, R. - Chambry, E. (trads.), (1966-1975): Plutarque. Vies, Paris, Les Belles Lettres.

Fromentin, V. - Schnäbele, J. (TRAD.), (1990): Denys d'Halicarnasse. Antiquités romaines, Les Belles Lettres, Paris.

Garnsey, P. (1970): Social status and legal privilege in the Roman empire, Oxford.

Glare, P. G. W. (1982): Oxford Latin Dictionary, Oxford.

Goelzer, H. (TRAD.), (1921): Tacite. Histoires, Paris, Les Belles Lettres.

Harmand, J. (1967): L'armée et le soldat à Rome de 107 à 50 avant J-C, Paris.

Hinard, F. (1993): "Sacramentum", Athenaeum 81, 252-263.

Hulot, H. - Berthelot, J. F. -Tissot, P.-A. - Bérenger, A. (trad.), (1979): Justinien. Les 50 livres du Digeste ou des Pandectes de l'empereur Justinien, Paris, Scientia.

$\mathrm{JAL}, \mathrm{P}$.

(1962): "Le soldat des guerres civiles à Rome à la fin de la République et au début de l'Empire", Pallas 11, 7-27.

(1963): La guerre civile à Rome. Étude littéraire et morale, Paris.

JAL, P. (TRAD.)

(1942): Tite Live. Periochae, Paris, Les Belles Lettres.

(2005): Tite Live. Histoire romaine, Paris, Les Belles Lettres.

Le Bohec, Y. (2004): “Desertor”, [en] H. Cancik - H. Schneider - M. Landfester (eds.), Brill's New Pauly: Encyclopaedia of the Ancient World. Cyr - Epy, Leiden - Boston - Cologne, 2004, pp. 315.

Marache, R. (TrAd.), (1989): Aulu-Gelle. Les nuits attiques, Paris, Les Belles Lettres. 
Pédech, P. (TRAD.), (1969): Polybe. Histoires, Paris, Les Belles Lettres.

Phang, S. E. (2008): Roman Military Service. Ideologies of Discipline in the Late Republic and Early Principate, Cambridge.

Reydellet, M. (TRAD.), (1984): Isidore de Séville. Etymologies, Paris, Les Belles Lettres.

Savagner, M. A. (Trad.), (1846): Festus. De la signification des mots, Paris, Bibliothèque latine-française.

Sheldon, R.-M. (2009): Renseignement et espionnage dans la Rome antique, Paris.

VAllejo Girvés, M.

(1993): "Sobre la persecución y el castigo a los desertores en el ejército de Roma", Polis $5,241-251$.

(1996): "La legislación sobre los desertores en el contexto político-militar de finales del siglo IV y principios del V d.C.”, Latomus 55, 31-47.

(1996a): "Transfugae en el ejército de Roma", Hispania Antiqua 20, 399-408.

Velho, G. (2002): "Les déserteurs des armées civiques en Grèce ancienne ou la négation du modèle du citoyen-soldat", Etudes classiques 70, 239-256.

Wolff, C. (2009): Déserteurs et transfuges dans l'armée romaine à l'époque républicaine, Napoli. 